\title{
SEPARATION OF COLLECTION CONCERN
}

\begin{abstract}
Separation of concerns is a common way to identify, encapsulate and manipulate only parts of software that are relevant to a particular concept, goal or purpose. Collection is an important concern of the programs, but it is mixed with data manipulation in the current programming languages. In addition, the current approach to handle collections are limiting the composition of collections. This paper introduces a novel approach called metastruct to encapsulate collection concern. The metastruct has sections to introduce various parts of a collection and operators to build a collection efficiently. The other feature of the metastruct mechanism is composition. It provides the ability to create composed metastructs based on others. Efficiency, simplicity and flexibility are the major features of the proposed approach.
\end{abstract}

Keywords: Programming Language, Collection Handling Concern, High-Level Abstraction, Collections Composition, Collections Efficiency 


\section{Introduction}

Separation of concerns is a common way to modularization. Generally, it refers to the ability to identify, encapsulate and manipulate only parts of software that are relevant to a particular concept, goal or purpose [1]. Concerns are the most primary motivation for organizing and decomposing any software into manageable and comprehensible parts [2]. Typical concerns in software include object [3], aspect [4], feature [5] and role [6]. To make evolving the code easier, software engineers generally try to keep concerns separate as they build a system [7]. Unfortunately, due to the limitations that programming languages impose on the decomposition of software systems, it is not possible to separate all concerns in software [8], [9]. So, in almost all programming languages one of the concerns is privileged at the expense of others. This is sometimes called the tyranny of the dominant decomposition [9].

An important concern of programs is collections. They are the building blocks of any program. They provide efficient mechanisms to store and retrieve a program's data [10]. Most programs use a variety of collections. They often use simple variations or compositions of basic collections [11] such as linked lists, queues, stacks and tree types. For example, Hadoop, a distributed processing framework for large data sets, uses many Java collections such as List , LinkedList, Queue, Set, TreeSet, LinkedHashSet, HashSet and HashMap. So, their efficiency has a significant effect on the overall of applications performance.

The common approaches such as generic programming and generalization to handle collections that is used in the current programming languages, have shortcomings. Some of them are: (i) they limit the performance of collections and (ii) they offer insufficient support of composing collections. In these approaches, encapsulation of the collection is accompanied by losing of the performance, thus establishing a trade-off between reusability and performance: libraries of collections versus hand-coded implementation [12]-[14]. As an example, hand-coded implementation of lists is more efficient than lists provided in libraries. Clearly, the success of the collection separation depends on its efficiency. Without an efficient model of integrating collection and data concerns, the separation is completely useless. So, programmers have to choose between them, and use the hand-coded implementation to reach more efficiency as possible. The hand-coded implementations have higher complexities and a larger volume of codes, increasing the costs of production and maintenance. Moreover, these efforts are often laborious and error-prone. Finally, in the hand-coded, collections concern end up to be scattered and tangled throughout the code of a program, so their reusability and extensibility are limited.

As an example, code snippet in Fig. 1 shows parts of the Linux inode. The inode is a collection in a Unix-style file systems that describes a file system object such as a file or a directory. It has two categories of fields. The first category is those that define the inode itself. Some of them are $i$ mode (used to indicate the format of the described file and the access rights), $i$ uid (user id associated with the file). The second category are those that are used to store the inode in some collections. Some of them are $i \_i o \_l i s t, i \_l r u$ and $i \_s b \_l i s t$. For example, $i_{-} s b \_l i s t$ is used to maintain the list of the inodes of a super block. Clearly, they are not related to the inode itself. The inode as a data element is an independent concern from lists that store it. But, for better performane, inode and lists end up to be tangled in the implementation. On the other hand, the specifications and operations of the lists collections are scattered in the code which makes tracing them a hard and boring task. Scattered concerns make it difficult for developers to reason about which pieces of code implements a concern. 
Besides, the way different concerns interact with each other is unclear. It reduces traceability, maintainability and reusability [9], [15].

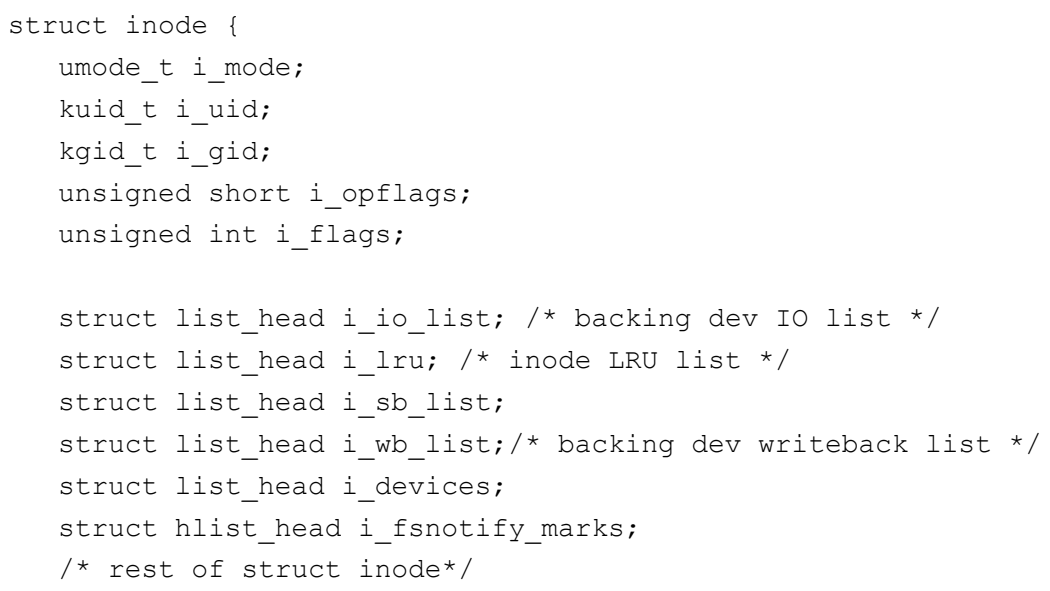

Fig. 1. The Linux inode

Another aspect of the collections implementation is the ability to compose primitive collections which leads to composite collections. Imagine that you need to create a collection that consists of two separate elementary structures (think of a phone-book with two indexes). The conventional solution is to store the elements only once and create a second structure (index) with pointers to the elements. Even if we ignore the run-time inefficiencies that this solution introduces, the programmer will have to maintain the collection on her own, and the common approaches do not provide any solution to the composition problem. [11], [17]. Composability of collections is closely coupled to evolvability, comprehensibility and modularity.

Efficient implementation of collections and separating collection concern are desired mechanisms for improving the quality of collections [7]. Separation of collections concern from other concerns reduces the software complexity during development, maintenance and reuse [3]. Requirements of separation of collection concern are [18]:

1. Independent Encapsulation: In general, encapsulation is the inclusion of one thing within another thing so that the included thing is not apparent. Encapsulation is a technique for minimizing interdependencies among separately-written modules by defining strict external interfaces.

2. Evolutionary Construction: Evolutionary construction refers to the capacity of the basic units to shape the selective environments such that they need. It requires new means to select the basic units and their composition to make new construction. Composable encapsulation solve these problems in a simple and elegant way [19].

Clearly efficient collections encapsulation and their flexible composition to reach the separation of collection concern are important requirements. To meet these requirements, this paper introduces a novel method called metastruct. The metastruct abstraction encapsulate the collection concern and, provides composition operators to support flexible composition of metastructs. The composition operators makes it possible that a composite metastruct can be made using other metastructs. 


\section{Metastruct Abstraction}

Examining collections shows that they have the same pattern. They have two components. The first one is a component, called root, to store the general information of the structure. The root is the entry point of the structure. It has reference(s) to some of the nodes of the structure.

The second one, called node, keeps the data elements. Every node has a data element and possibly some references to other nodes. The node usually does not perform separate operations except for providing the data.

A metastruct is an abstraction that encapsulates a collection including the implementation of its root, node and operations. It is independent from the data which is stored in it. Like a Java class, it has some attributes and methods to define the behaviors. However, it has a special section called element to define the node. This section is a hypothetical type, element, which is used to define variables and parameters inside a metastruct. However, it is not possible to create an object of element type.

Like Java, a metastruct operation has a pointer, this, to the metastruct itself. But unlike Java nested classes that have some hidden pointers to provide accessibility to the parent class, the metastruct does not have any additional pointers. Therefore, operations of the element section do not have any access to root section and vice versa. To clarify, code snippet in Fig. 2 defines metastruct LinkedList. The element section defines the LinkedList node. The remove operation takes an argument of type element and removes it from the list.

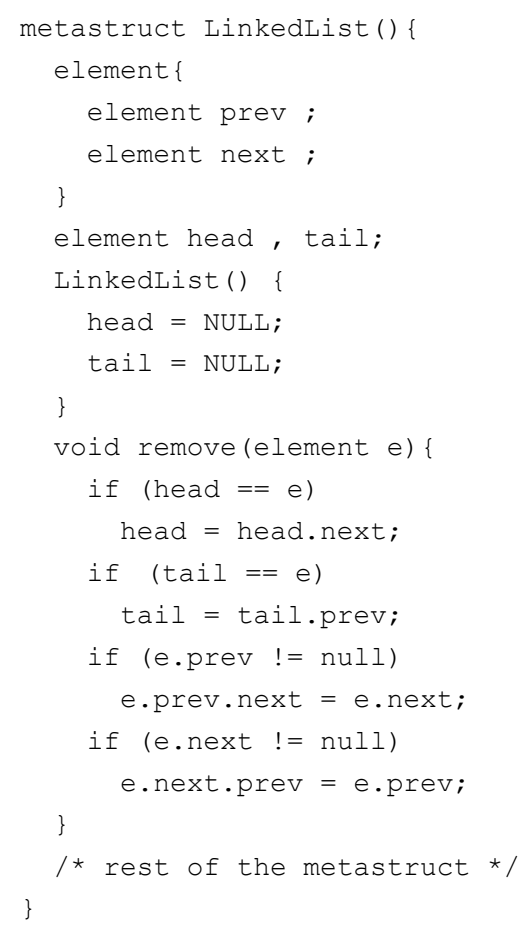

Fig. 2. Metastruct LinkedList 
Unlike conventional collections, a metastruct cannot be instantiated directly. Instantiation of a metastruct is done via applying it on a data set. As an example of applying a metastruct, assume that class Person is defined as in Fig. 3.

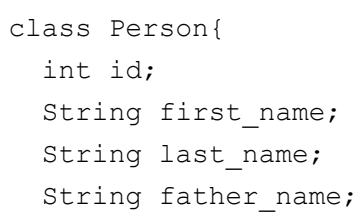

Fig. 3.Class Person

The following declaration defines a LinkedList of Person objects.

Person [LinkedList() ] people;

When a metastruct is applied on a data set, its data type is mapped to the element of the metastruct. Therefore, the hypothetical type (element) is mapped to the actual data type. In the above example, the element is mapped to class Person. Therefore the operations of the LinkedList operate on class Person. Now if $p$ is an object of class Person, it can be removed from people as follow:

people.remove (p) ;

\subsection{Key}

An important characteristic of collections is key values. To support key values, the metastruct has a special mechanism: hypothetical key type. If a metastruct has a key, it should define a key type. Inside a metastruct, the key type is like a usual data type. It can be used to declare variables and arguments. The only attribute of a key type is that it defines a linear order on the elements of the data set. Therefore, it is possible to compare two key values by their key.

Fig. 4.Metastruct Tree

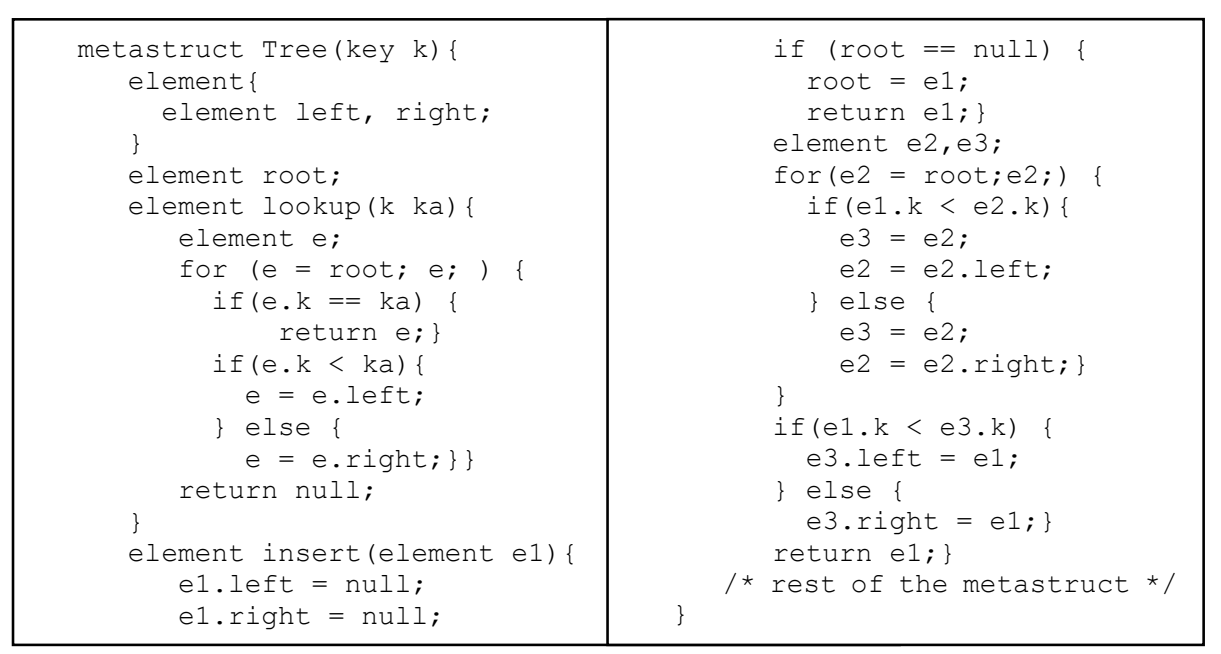

Often it is required to extract the key of a data element. Assume that $e$ is a data element that the key $k$ is defined on it, e.k extracts it. As an example of key type, Fig. 4 has the 
definition of the binary search tree metastruct (Tree). It shows that metastruct Tree has a key type named $k$. The lookup operation has an argument of type $k$ and finds an element having that key, i.e. $e . k==k a$. Again in the body of insert operation $k$ used to compare two key values, $e 1 . k<e 2 . k$. A metastruct can have more than one key. As shown in the following code snippet, $k 1$ and $k 2$ are defined as two key types of $X$.

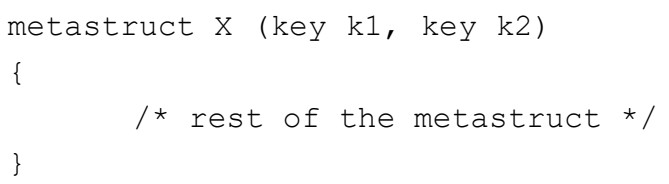

When a metastruct is instantiated, its abstract key types should be assigned values. The value of a key is a sequence of expressions composed of the data element attributes and literals. The definition of an expression of a key type is embraced in a $<$ pair. Some examples of key definitions are followed (Person is the base type):

$\langle i d\rangle$

$<$ lname, fname $>$

The first expression consists of one attribute and the second one consists of two attributes. For instance, applying the metastruct Tree can be done as follows:

Person [Tree $(<i d\rangle)$ ] people;

\section{Metastruct Composition}

Metastruct composition is used not only to create new metastructs from the available ones but also to apply multiple metastructs on a set of data elements. It gives complete control over all aspects of the composition. It provides the ability to add new attributes or operations to each component of the composed metastruct.

A composed metastruct has a composition expression. The composition expression defines how the composed metastruct is made up from other metastructs. It is made from two elementary operators: horizontal composition and vertical composition.

The horizontal composition is used when more than one metastruct is applied on a set of data elements. It is similar to a database table which has more than one index. However, it is not necessary to have a data element in all components. A data element can be placed in one or all of them (Fig. 5). In the horizontal composition, the node of composed metastruct is formed by concatenating the elements of the component metastructs. The metastruct itself is composed by concatenating the component metastructs.

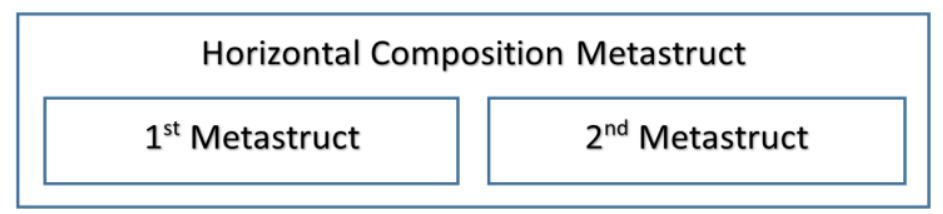

Fig. 5. Horizontal Composition of Metastructs

The code snippet in Fig. 6 provides $Q T$, the horizontal composition of Tree and LinkedList metastructs. LinkedList has operations to insert an element and delete an element efficiently. However, it does not have an operation to remove an element by key. To support it, the LinkedList can be composed with the Tree. In this composition every element is stored in both. To remove an element by its key, the Tree is used to find the element and then it is 
removed from the LinkedList and the Tree. Each node of the $Q T$ is a node of the LinkedList and Tree. Fig. 6 shows remove operation of the composed metastruct. It operates in $\mathrm{O}(\log n)$ time.

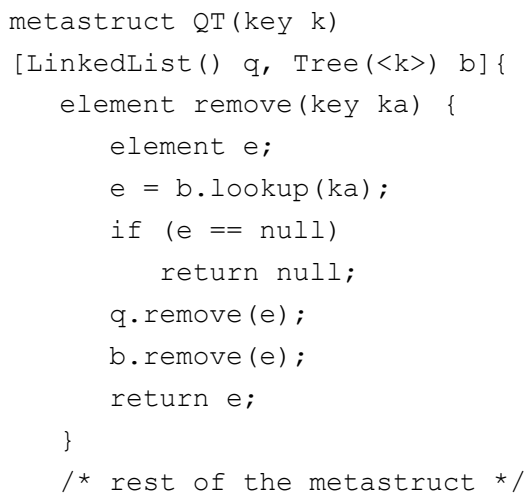

Fig. 6. Metastruct QT

The vertical composition happens when the elements of a collection are themselves collections. Fig. 7 shows parts of a vertical composition. The composition expression indicates that metastruct VCompos is a Tree whose nodes are LinkedList. A name, Node is assigned to the LinkeList in the composition expression to provide the capability of adding a new attribute, keynode to it in the body VCompos. The implementation provides one operation: insert. It calls the lookup operation of the Tree to get the corresponding LinkedList. Then, the insert operation of the LinkedList is called to insert the element.

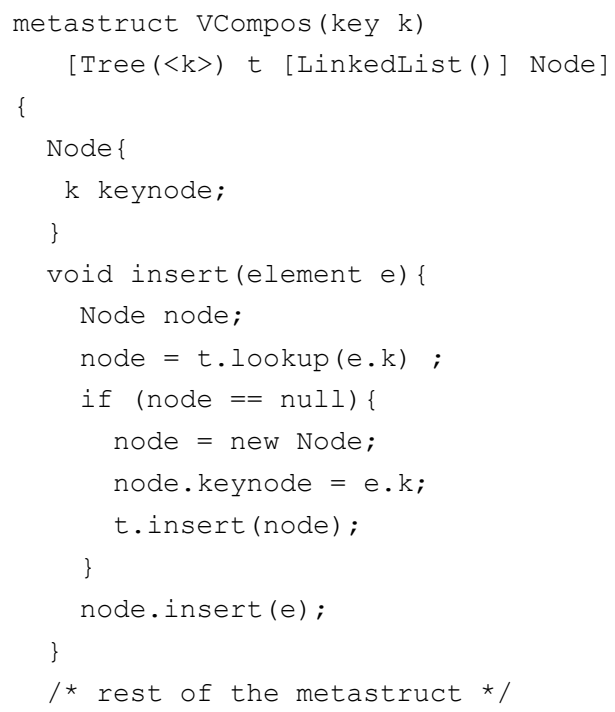

Fig. 7. Metastruct VCompos

In the above, VCompos has three level of structures: root, intermediate nodes and nodes (Fig. 8). At the first level, root, the root of Tree is placed. At the second level, intermediate 
nodes, the concatenation of Tree node and LinkedList root are placed. At the third level, nodes, the nodes of LinkedLists are placed.

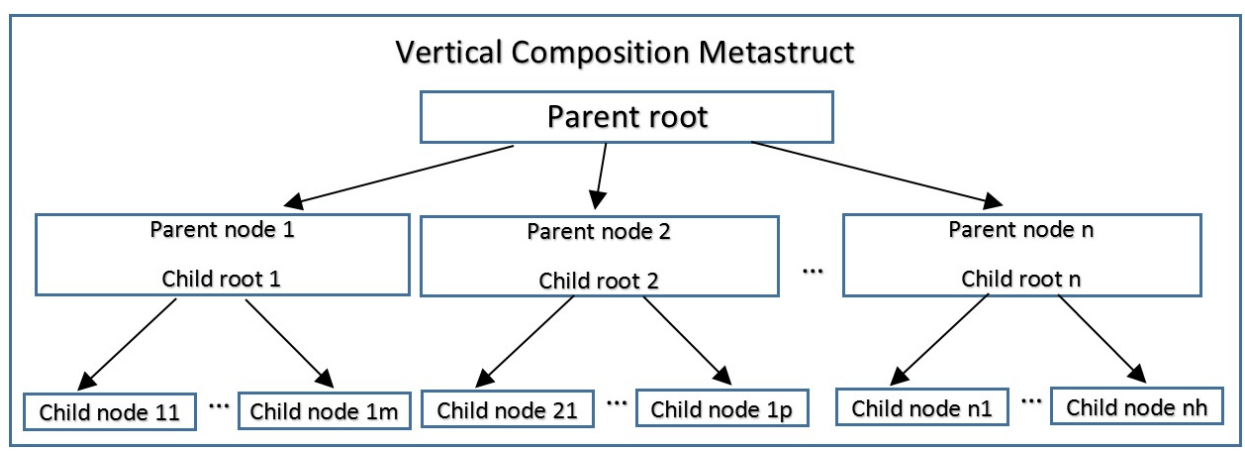

Fig. 8. Vertical Composition of Metastructs

Using horizontal and vertical operators, the composition expression is capable to make any collection from the primitive ones.

To show the power of composition expression consider the following example. In a data management system, a data record may be inserted in more than one indexes. A sample of this structure is shown in Fig. 9. In this collection, each data record is stored in a tree (or $\mathrm{B}^{+}-$Tree) based on its primary key (the left path in Fig. 9) and on a hash of linked lists and ordered lists (the right path in Fig. 9) based on the secondary key. Each linked list and ordered list has records with the same secondary key. When the records of a secondary key are needed, the corresponding linked list is retrieved. Also, when the list of a secondary key ordered by their primary key is needed, the corresponding ordered list is retrieved. The following code snippet shows the composition expression. Indexes gives capabilities of inserting, removing, and searching data in $\mathrm{O}(\log \mathrm{n})$ time.
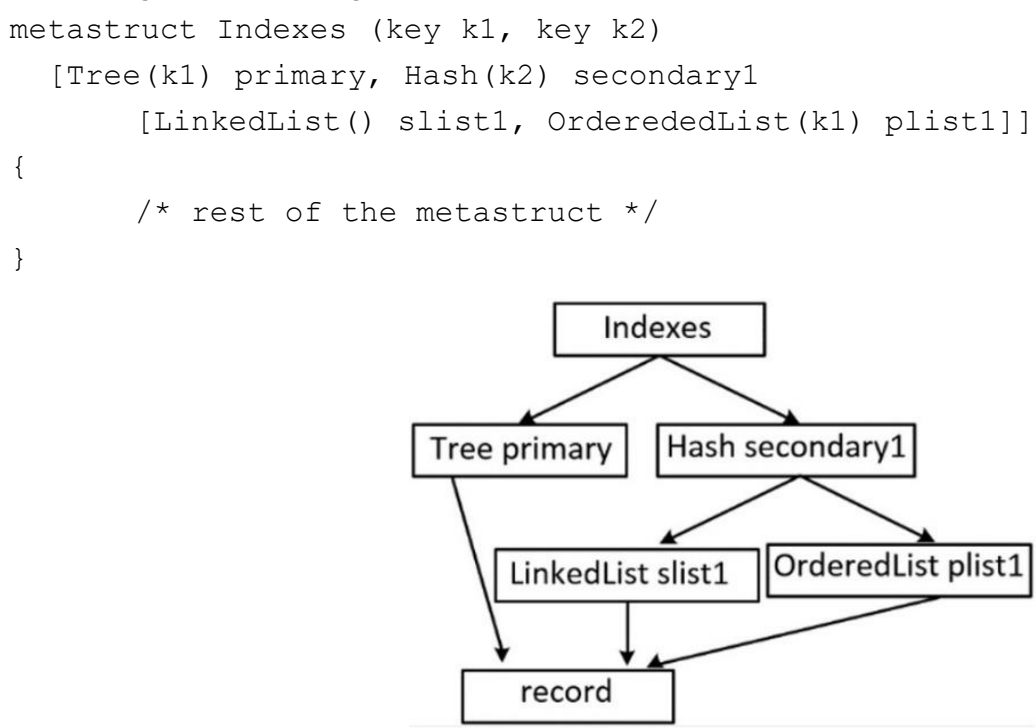

Fig. 9. The layout of a complex composition 
To achieve this goal, assigning a name to each component is provided in the grammar. Later on, inside the body of the composed metastruct, variables of that type can be declared, and new details can be added to it. Moreover, the implementation of new operations can be defined based on the components operations, or implemented independently.

\section{Implementation}

We have outlined a general, language-independent model of collections encapsulation and composition. In this section we have described a proof-of-concept for Java that demonstrates the feasibility of the idea. The metastruct was implemented as an extension to Java programming language. The compiler gets a code in the extended language and produces output in Java language. The output can be compiled using any Java compiler to produce byte code.

Fig. 10 shows abstract syntax of the metastruct. In the syntax, identifier is considered for displaying the terms which are arbitrarily selected by the programmer. The syntax starts with metastruct term (Metastruct) as the start symbol. Overall description of some non-terminals is as follows.

- Metastruct term (Metastruct): It presents an overall view of the metastruct consists of its name, keys, composition expression and body.

- Composition term (Compose): It is a sequence of horizontal composition which are vertically composed. The horizontal compositions are separated by comma. Each vertically composition term is embraced within a [ ] pair. A composition term can be a metastruct instance (ms_instance), horizontally composition or a vertical composition. ms_instance refers to the applying of one of the previously defined metastructs. The applying of a metastruct is done by calling it along with its parameters.

- Element Section (Element): It defines detail of node consists of fields and operations.

$$
\begin{aligned}
& \text { Metastruct } \left.::=\text { metastruct identifier ( }(k e y \text { identifier })^{*} \text { )[Composition] \{Element Field } * \text { Method } *\right\}
\end{aligned}
$$

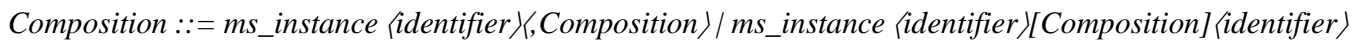

$$
\begin{aligned}
& \text { Element } \quad \because=\text { element }\{\text { Field } * \text { Method } * \text { \} } \\
& \text { Field } \quad: \because=\text { Type identifier } \\
& \text { Type } \quad::=\text { element } \mid \text { int } \mid \text { String } \mid \text { void } \\
& \text { Method } \left.\quad::=\text { Type identifier( }(\text { Type identifier }\rangle^{*}\right)\{\text { return }(\text { Exp })\} \\
& \operatorname{Exp} \quad \therefore=\text { identifier } \mid \text { Exp.identifier } \mid \text { Exp.identifier(Exp*) }
\end{aligned}
$$

Fig. 10. Abstract Syntax of the Metastruct

In the syntax (Fig. 10) angle brackets $\langle\ldots\rangle$ indicates that the item within the brackets is optional, superscript $*$ for repetition zero or more times. Sequences of key declarations, field declarations (Field) and operation declarations (Method) are assumed to contain no duplicate names. 
To achieve the flexibility, in the grammar, a name (identifier) can be assigned to each component of composition, especially to the intermediate nodes of vertical compositions. Later on, inside the body of the composed metastruct, variables of that type can be declared, and new details can be added to it. Moreover, the implementation of new operations can be defined based on the components operations, or independently.

\subsection{Translation to Java}

The compiler is implemented as a multi-pass translation to Java. The translation process is implemented by means of common tools such as JFlex and Cup. It includes three phases: lexical analysis, parsing and code generation (Fig. 11).

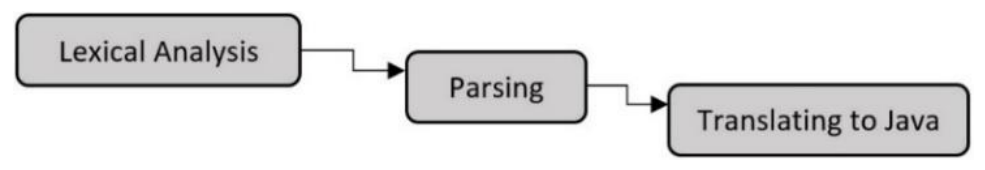

Fig. 11. Translation from the metastruct to Java

In the first stage of lexical analysis and parsing, syntactic checks like multiple declarations of the same named metastructs, or declaration of element sections and operations are done. If metastruct declaration and usage are matched, then the next step is the translation into Java. When we compile the back-end generated Java for execution, type checking is handled in Java. The translation into Java is the most demanding step. During this stage, structural translation rules are followed to translate each class and metastruct into one or multiple classes. One class is produced for every metastruct without composition. In horizontal composition, two classes are produced for everyone. In vertical composition, three class is produced for everyone. The resulting classes get together to build the complete Java representation of the source.

In current implementation a copy of code is produced for each instantiation. It was not compulsory, but Java do not allow to create intermediate pointer to structure. While in $\mathrm{C}$ or $\mathrm{C}++$ implementations it does not need a copy.

\subsection{Metastruct Layout}

When a metastruct is applied on a set of data elements, its element section is injected into the data elements. Assume that the LinkedList metastruct is implemented as is presented in Fig. 2. Consider the following code snippet, metastruct LinkedList is applied on class Person.

Person [LinkedList() ] people;

Fig. 12 shows the produced code for the above code snippet. As noted before, element part of LinkedList is injected to class Person as data element. So, there is no need to additional references to operate on collections. References to class Person are added to class people LinkedList as root of the collection. Also method remove is customized and added to class people_LinkedList based on class Person as data element. As the data element and the node of collection is concatenated together, so there is no need to scan collection, and its remove operation be in $\mathrm{O}(1)$ time. 


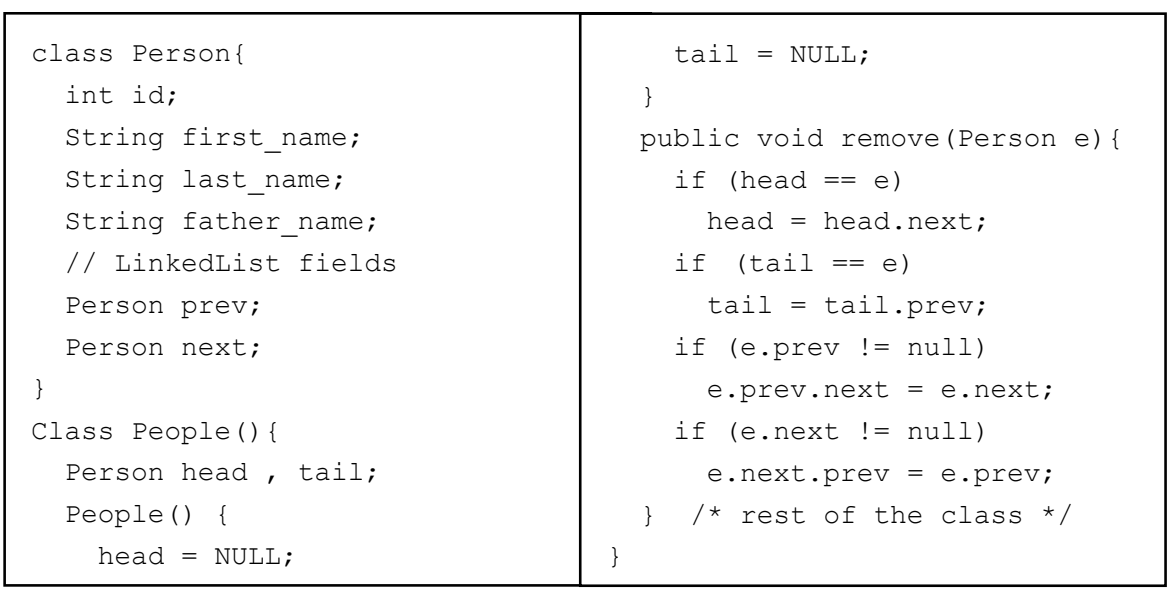

Fig. 12. Produced Code for Applied Metastruct

In the horizontal composition, the fields of the metastructs are concatenated to form the composed metastruct and the element sections of them are concatenated to form the node of the composed metastruct.

In the vertical composition, the first metastruct is called parent metastruct and the second one is called child metastruct. The root of the parent forms the root of the composed metastruct. The element section of the parent is concatenated to the root of the child to form the intermediate node. Finally, the element section of the child metastruct forms the element section of the composed metastruct.

In case of applying more than one metastructs on a set of data elements, the element sections of the metastructs are injected to the data element.

\section{Evaluation}

This section evaluates metastruct approach with respect to separation of collection concern on four properties: encapsulation, evolvability, reusability, and comprehensibility.

\subsection{Encapsulation}

Minimizing interdependencies among separately-written modules by defining strict external interfaces is the most important feature of encapsulation. In the metastruct, interdependencies are provided through composition expressions as a strict external interface. So, internal of metastructs do not affected by interdependencies of metastructs, and each metastruct can be implemented independently and then composed together.

\subsection{Evolvability}

Systems are known to continuously evolve while they are being developed and used in order to cope with changing requirements. On the one hand the fact that the composition is specified outside of the metastruct and can be changed should mean that it is easier to cope with independently evolving metastructs that are used in a composition. As an example, assume that at a certain point in time we have implemented the example as Section 3. It can be seen there that a number of metastructs is taken together and composed. The composition uses all of 
our features to implement the desired semantics. When one of the metastructs, or the base code, evolves we can make a new composition that again expresses the desired semantics.

\subsection{Reusability}

One of the main goals of separation of concerns is to increase reuse. Indeed, collections allow base code to be reused in several programs. Besides base code one also can ponder the question of reusing collections in different compositions, which is indeed the context of this paper. Metastruct abstraction provides a new approach to implement data-independent and program-independent implementation of collections. A prepared metastructs library that can be easily applied to a variety of applications is leading to reusability. Moreover, composition mechanism provides the ability that a metastruct is made efficiently using other metastructs; it will truly improve reusability to a new level, because it makes it easier to compose collections and the semantics of the composition is expressed outside of the collections themselves. The price to pay for this increased reuse promise is to learn and use yet another expression besides the collections. Additionally, composition expression provides flexible control over all aspects of the composition. All of these features contribute to the efficiency, flexibility and configurability of metastructs.

\subsection{Comprehensibility}

The goal of metastruct approach is to improve comprehensibility of collections compositions by using a composition approach. The approach does not make any decision on behalf of the programmer. It is based on simplicity and flexibility. Moreover the main concepts of our proposal are supposed to be resolve fairly concrete problems and not overlap, similar to the zero-one-infinity principle in programming language design. We have not done experiments to verify if our approach is indeed comprehensible or not.

\section{CONCLUSION}

This paper introduced a new approach to implement collections. The approach introduces a new abstraction, the metastruct to define a collection, a new approaches to define key attributes and two operators to build composite collections. The metastruct approach is the first step towards the separation of collections concern and provides its encapsulation and evolvability requirements. Based on separating collection handling concern, the approach creates a program-independent way to the definition and manipulation of collections.

We have outlined a general, language-independent model of collections encapsulation and composition, and we have described a proof-of-concept for Java that demonstrates the feasibility of the idea. The approach can be incorporated into a language in such a way that there is an additional precompilation only when one wishes to make use of the approach. In other cases, default libraries mimic the conventional approach offered by the language.

The approach does not make any decision on behalf of the programmer. It is based on simplicity and flexibility. Moreover, high reusability and low maintenance cost are other advantages of the metastruct approach. The provided case study and other samples show that the approach effectively decreases the volume of code and the runtime complexity.

\section{References}

[1] W. L. Hürsch and C. V. Lopes, "Separation of concerns," Tech. report, North East. 
Univ., 1995.

[2] H. Ossher and P. Tarr, "Multi-Dimensional Separation of Concerns and the Hyperspace Approach," in Software Architectures and Component Technology, 2002, pp. 293-323.

[3] S. Jarzabek and K. Kumar, "On Interplay between Separation of Concerns and Genericity Principles : Beyond Code Weaving," Comput. Sci. Inf. Syst., vol. 13, no. 3, pp. 731-758, 2016.

[4] A. Kumar, A. Kumar, and M. Iyyappan, "Applying Separation of Concern for Developing Softwares Using Aspect Oriented Programming Concepts," Procedia Comput. Sci., vol. 85, pp. 906-914, 2016.

[5] J. Kienzle, G. Mussbacher, P. Collet, and O. Alam, "Delaying decisions in variable concern hierarchies," ACM SIGPLAN Not., vol. 52, no. 3, pp. 93-103, 2017.

[6] E. P. Andersen and T. Reenskaug, "System design by composing structures of interacting objects," in ECOOP 1992: ECOOP '92 European Conference on Object-Oriented Programming, 1992, p. pp 133-152.

[7] D. L. Parnas, "On the criteria to be used in decomposing systems into modules," Commun. ACM, vol. 15, no. 12, pp. 1053-1058, 1972.

[8] G. Kiczales et al., "Aspect-oriented programming," in ECOOP 1997: European Conference on Object-Oriented Programming, 1997, p. pp 220-242.

[9] P. Tarr, H. OSSHER, W. HARRISON, and S. M. SUTTON, "N degrees of separation: Multi- dimensional separation of concerns," in The 21st International Conference on Soft-ware Engineering, 1999, pp. 107-119.

[10] A. C. Fleck, "Recent developments in the theory of data structures," Comput. Lang., vol. 3, no. 1, pp. 37-52, 1978.

[11] D. Batory, V. Singhal, M. Sirkin, and J. Thomas, "Scalable software libraries," in SIGSOFT '93 Proceedings of the 1st ACM SIGSOFT symposium on Foundations of software engineering, pp. 191-199.

[12] J. Yallop, "Staged generic programming," Proc. ACM Program. Lang., vol. 1, no. ICFP, p. 29, 2017.

[13] C. Loncaric, "Cozy : Synthesizing Collection Data Structures," in Proceedings of the 2016 24th ACM SIGSOFT International Symposium on Foundations of Software Engineering, 2016, pp. 1103-1105.

[14] R. Singh and A. Solar-Lezama, "Synthesizing data structure manipulations from storyboards," in Proceedings of the 19th ACM SIGSOFT symposium and the 13th European conference on Foundations of software engineering, 2011, pp. 289-299.

[15] J. Dolby and A. Chien, "An automatic object inlining optimization and its evaluation," ACM SIGPLAN Not., vol. 35, no. 5, pp. 345-357, 2000.

[16] Y. Smaragdakis and D. S. Batory, "DiSTiL: A transformation library for data structures," in Proceedings of USENIX Conference on Domain-Specific Languages, 1997, no. October, p. 257270.

[17] P. Hawkins, M. Rinard, A. Aiken, M. Sagiv, and K. Fisher, "An introduction to data representation synthesis," Commun. ACM, vol. 55, no. 12, p. 91, 2012.

[18] N. Schärli, S. Ducasse, O. Nierstrasz, and R. Wuyts, "Composable encapsulation policies," in ECOOP, 2004, pp. 26-50. 
[19] A. Marot and R. Wuyts, "Composability of aspects," in Proceedings of the 2008 AOSD workshop on Software engineering properties of languages and aspect technologies, 2008, p. 5.

Davud Mohammadpur received his B.Sc. degree in Software Engineering from Kharazmi University, Tehran, Iran. He received his M.Sc. degree in Software Engineering from Iran University of Science and Technology, Tehran, Iran. He is currently a Ph.D. student at the Department of Information and Communication Technology(ICT), Malek-Ashtar University of Technology, Tehran, Iran.

Ali Mahjur received his $\mathrm{PhD}$ from Sharif University of Technology. Currently he is a faculty member of Malek Ashtar University of Technology. His research interests are programming languages, operating systems and processor microarchitecture 